\title{
Neurovascular Protective Effect of FeTPPs in N-Methyl-D-Aspartate Model
}

\section{Similarities to Diabetes}

\author{
Mohammed M.H. Al-Gayyar, ${ }^{\text {†† }}$ \\ Mohammed A. Abdelsaid, ${ }^{\star \dagger}$ \\ Suraporn Matragoon, ${ }^{\star \dagger}$ Bindu A. Pillai, ${ }^{* \dagger}$ \\ and Azza B. El-Remessy*†§प \\ From the Program in Clinical and Experimental Therapeutics," \\ College of Pharmacy, University of Georgia, Augusta, Georgia; \\ Charlie Norwood Veterans Affairs Medical Center, ${ }^{\dagger}$ Augusta, \\ Georgia; the Department of Biochemistry, ${ }^{\ddagger}$ Faculty of Pharmacy \\ University of Mansoura, Mansoura, Egypt and the Departments \\ of Pharmacology and Toxicology, ${ }^{\S}$ and Ophthalmology, "Tedical \\ College of Georgia, Augusta, Georgia
}

We have previously shown a causal role of peroxynitrite in mediating retinal ganglion cell (RGC) death in diabetic and neurotoxicity models. In the present study, the role of peroxynitrite in altering the antioxidant and antiapoptotic thioredoxin (Trx) system will be investigated as well as the subsequent effects on glial activation and capillary degeneration. Excitotoxicity of the retina was induced by intravitreal injection of $N$-methyl-Daspartate (NMDA) in rats, which also received the peroxynitrite decomposition catalyst FeTPPs. RGC loss was assessed by terminal deoxynucleotidyl transferasemediated dUTP nick-end labeling and GC count. Glial activation and nitrotyrosine were assessed by immunohistochemistry. Acellular capillaries and pericytes were counted in retinal trypsin digest. NMDA-induced peroxynitrite formation caused RGC loss, which was associated with enhanced expression of Trx and its endogenous inhibitor thioredoxin interacting protein. The results also showed enhanced thioredoxin interacting protein/Trx binding and disruption of the Trx/apoptosis signal-regulating kinase 1 "inhibitory complex," leading to release of apoptosis signal-regulating kinase 1 and activation of the apoptotic pathway, as evidenced by 38 MAPK and poly-ADP-ribose polymerase activation. Furthermore, NMDA caused glial activation and compromised retinal vasculature, as indicated by acellular-capillary formation and pericyte loss. Treatment with FeTPPs blocked these effects. In conclusion,
NMDA-induced retinal neuro/vascular injury is mediated by peroxynitrite-altered Trx antioxidant defense, which in turn activates the apoptosis signal-regulating kinase-1 apoptotic pathway. In addition to acute RGC death, an NMDA model can be a useful tool to study glial activation and capillary degeneration in retinal neurodegenerative disorders, including diabetic retinopathy. (Am J Patbol 2010, 177:1187-1197; DOI: 10.2353/ajpath.2010.091289)

Retinal ganglion cell (RGC) death plays a critical role in the pathogenesis of many retinal neurodegenerative disorders, including glaucoma, diabetic retinopathy, traumatic optic neuropathy, uveitis, and retinal ischemia. ${ }^{1-5}$ Understanding the molecular mechanism of neuronal cell death in such retinal diseases is of great clinical important for devising new treatments. In response to traumatic or ischemic injury, excessive activation of glutamate receptors has been implicated in the RGC death process. ${ }^{6}$ Therefore, administration of N-methyl-D-aspartate (NMDA) to rodent eyes is a reliable model to study RGC death. Excessive NMDA receptor stimulation leads to excessive $\mathrm{Ca}^{2+}$ influx, ${ }^{7}$ which in turn triggers formation of nitric oxide ${ }^{8}$ and accumulation of superoxides and their combination product peroxynitrite, causing lipid peroxidation, DNA damage and the eventual cell death. ${ }^{9}$ Although increases in reactive oxygen species (ROS) and peroxynitrite have been implicated in mediating RGC death, ${ }^{1,6}$ the molecular mechanisms by which peroxynitrite causes RGC death are not fully elucidated.

Protection from ROS is mediated by superoxide dismutase, glutathione, and thioredoxin (Trx) systems. ${ }^{10,11}$

Supported by Career Development grant 2008-149 from Juvenile Diabetes Research Foundation, Scientist Development grant from the American Heart Association.

Accepted for publication May 20, 2010.

Address reprint requests to Azza B. El-Remessy, Ph.D., R.Ph., FAHA, Clinical and Experimental Therapeutics, College of Pharmacy, University of Georgia, Augusta, GA 30912. E-mail: aelremessy@mcg.edu. 
The Trx system is a ubiquitous thiol-reducing system that includes thioredoxin $(12 \mathrm{kDa})$, thioredoxin-interacting protein (TXNIP), homodimeric seleno-protein thioredoxin reductase, and NADPH. ${ }^{12}$ Trx is not only an antioxidant, but it can also regulate cell survival by binding and inhibiting the activity of apoptosis signal-regulating kinase 1 (ASK-1), a recently identified member of MAP kinase kinase kinase (MAPKKK) family. ${ }^{13}$ While the activity and expression of thioredoxin is regulated by TXNIP, also known as vitamin-D3 up-regulated protein-1, the expression of TXNIP is directly regulated by $\mathrm{Ca}^{2+}$ influx. ${ }^{14}$ In addition to modulation of cellular redox state, TXNIP plays a critical role in stress-induced cellular apoptosis as it binds reduced Trx and inhibits its activity. Thus, under stress conditions, Trx can dissociate from ASK-1 and gain a kinase activity to activate JNK and p38 MAPK signaling pathway leading to apoptosis. ${ }^{13,15} \mathrm{How}$ ever, the expression of TXNIP and its interaction with Trx-ASK-1 complex to mediate RGC death in the NMDA model remains unknown.

The activation of glial cells, demonstrated by increased glial fibrillary acidic protein staining, has been well documented in response to neuronal injury including NMDA model. ${ }^{16,17}$ Additional studies showed that NMDA increased the expression of proinflammatory cytokines, endothelial adhesion molecules, and leukocyte recruitment. ${ }^{18}$ However, There is a gap in our knowledge about the vascular changes that might follow the neuronal damage in models of optic neuropathy. The research has been focused on the neuronal aspect of optic neuropathy but not on the long term vascular changes. Therefore, this study aims to examine the long-term effects on the vasculature after neuronal cell death and glial activation in the NMDAinjected rat model. Furthermore, the role of peroxynitrite in altering the interaction between Trx, TXNIP and ASK-1 leading to apoptosis will be explored using the specific peroxynitrite decomposition catalyst (FeTPPs).

\section{Materials and Methods}

\section{Animals}

All procedures with animals were performed in accordance with the ARVO Statement for the Use of Animals in Ophthalmic and Vision Research and the Charlie Norwood VA Medical Center Animal Care and Use Committee. Four sets of animals were prepared for a total of 60 male Sprague Dawley rats ( $\sim 250 \mathrm{~g}$ body weight) that were divided into four groups: one group received a single intravitreal injection of NMDA (40 nmol, Sigma, St. Louis, MO), the second group received a single intravitreal injection of both NMDA (40 nmol) and 5,10,15,20-tetrakis(4-sulfonatophenyl) porphyrinato iron (III) (FeTPPs, $100 \mu \mathrm{g} / \mathrm{eye}$, Calbiochem, CA), the third group was intravitreally injected with $\mathrm{N}$-methyl-Laspartate (NMLA, $40 \mathrm{nmol}$, Sigma) and served as a control group, and the last group was intravitreally injected with both NMLA (40 nmol) and FeTPPs (100 $\mu \mathrm{g} / \mathrm{eye})$ to serve as treated control group. The dose of NMDA was selected based on a previous study ${ }^{19}$ and the dose of FeTPPs was selected based on our previous study ${ }^{1}$ similar to the dose of
NOS inhibitor. Treatment with NMDA or FeTPPs did not alter rats' body weight during 1 week of treatment (data not shown).

\section{Intravitreal Injection}

Intravitreal injection of NMDA was performed as previously described by our group ${ }^{1}$ with minor modification. Shortly, animals were deeply anesthetized by intraperitoneal injection of pentobarbital $(35 \mathrm{mg} / \mathrm{kg})$. Before intravitreal injection, pupil dilator (atropine) and ophthalmic topical anesthetic (Marcaine) were applied topically on rat eyes. Rats received a single intravitreal injection of one of the treatment solutions using 30-gauge needles mounted to $10-\mu \mathrm{l}$ Hamilton syringes. The volume of intravitreal injections was set at $4 \mu \mathrm{l}$, a volume that does not cause eye damage as described before by our group. ${ }^{1}$ To ensure the proper delivery and even distribution of the intravitreally injected compounds, all solutions for intravitreal injection contained $5 \mu \mathrm{g} / \mathrm{ml}$ of Fast Green FCF (Sigma). Neomycin/polymyxin-B/Bacitracin ophthalmic ointment was applied to the injected eyes and animals were allowed to recover. Rats were sacrificed after 1 day (short term) or 7 days (long term) of injection and the eyes were enucleated and processed for further analyses.

\section{Evaluation of Neuronal Cell Death in Rat Retina}

Terminal deoxynucleotidyl transferase-mediated dUTP nickend labeling (TUNEL) was performed using the ApopTAG in situ apoptosis detection kit fluorescein (Millipore, CA), following the manufacturer's directions. Briefly, OCT-frozen eye sections $(10 \mu \mathrm{m})$ from each group were fixed using paraformaldehyde and ethanol:acetic acid (2:1). Then, the samples were incubated with terminal deoxynucleotidyl transferase enzyme followed by incubation with anti-digoxigenin conjugate. Propidium iodide, $1 \mu \mathrm{g} / \mathrm{ml}$, was added as a nuclear counterstain. On completion of the TUNEL assay, cover slips were applied using Vectashield mounting medium for fluorescence (Vector Laboratories). Each section was systematically scanned for positive green fluorescent cells in retinal layers indicating apoptosis. Images were obtained using an AxioObserver.Z1 microscope (Zeiss, Germany) with 200× magnification. Five to seven animals were used for each group and the number of TUNEL positive cells were counted in four fields of different areas of each retina and calculated as the number $/ \mathrm{mm}^{2}$ of retinal area.

\section{Counting Number of Neuronal Cells in the Ganglion Cell Layer}

OCT frozen retinal sections were stained with hematoxylin and eosin for light microscopy. The nuclei in the ganglion cell layer (GCL), not including nuclei in the vessels were counted in four locations in the retina (both sides of the optic nerve (posterior) and mid-retina (central) as described previously. ${ }^{2}$ Four animals from each group and two fields for each location were used. The number of nuclei was calculated per $\mathrm{mm}^{2}$ of retinal area. In addition, fixed retina sections reacted with monoclonal anti-Brn-3a 
antibody (specific RGC marker, Santa Cruz) followed by Texas red-conjugated goat anti-mouse antibody (Molecular Probes, OR). Data were analyzed using AxioObserver.Z1 Microscope (Zeiss, Germany).

\section{Immunohistochemistry}

The distribution and quantification of ASK-1 and nitrotyrosine expression as well as glial cells activation were analyzed in retinal sections. OCT-frozen sections $(10 \mu \mathrm{m})$ of eyes were fixed using $2 \%$ paraformaldehyde solution in PBS for 10 minutes. Sections were washed several times with PBS and were reacted with monoclonal anti- glial fibrillary acidic protein (GFAP) antibody (for glial cells activation, Affinity BioReagents), polyclonal ASK-1 antibody (Santa Cruz, CA) or polyclonal anti-nitrotyrosine antibody (Calbiochem, CA) followed by Texas red-conjugated goat anti-mouse antibody or Oregon green-conjugated goat anti-rabbit antibody (Molecular Probes, OR). Data (three fields/retina, $n=6$ in each group) were analyzed using AxioObserver.Z1 microscope (Zeiss, Germany) and AxioVs 40 V4.7.2.0 software to quantify the density of immunostaining. Lectin stain for colocalization of blood vessels with TUNEL-positive cells was done by incubating fixed retina sections $(10 \mu \mathrm{m})$ in isolectin $\mathrm{B}_{4}$ over night followed by Texas red avidin D (Vector, CA) as described previously by our group. ${ }^{20}$

\section{Quantitative PCR (qPCR)}

Retinal mRNA was prepared according to the manufacturer's instructions as described in our recent study. ${ }^{3}$ The OneStep qRT-PCR Invitrogen kit was used to amplify $10 \mathrm{ng}$ of retinal mRNA from each sample. PCR primers were designed to amplify thioredoxin: 5'-GCCAAAATGGTGAAGCTGAT-3' and reverse primer 5'-TGATCATTTTGCAAGGTCCA-3'. TXNIP primers 5'-AAGCTGTCCTCAGTCAGAGGCAAT-3' and reverse primer 5'-ATGACTTTCTTGGAGCCAGGGACA-3'. Amplification of 18S rRNA was used as an internal control. Quantitative PCR was performed using a Realplex Mastercycler (Eppendorf, Germany). Trx and TXNIP expression was normalized to the $18 \mathrm{~S}$ level in each sample and expressed as relative expression to normal controls.

\section{Western Blot Analysis and Immunoprecipitation}

Rat retinas were isolated and homogenized in radioimmunoprecipitation assay buffer as described previously. ${ }^{1}$ The total amount of protein was determined by protein assay (Bio-Rad, CA). Samples (50 $\mu$ g protein) were separated by SDS-PAGE and electroblotted to nitrocellulose membrane, and the membranes were blocked in Tris-buffered saline, $0.02 \%$ Tween 20, containing 5\% nonfat milk. Antibodies for Trx, TXNIP, ASK-1 (Santa Cruz, CA), p-p38, p38 (Cell Signaling, MA) and poly-ADP-ribose polymerase (PARP) (BD PharMingen) were applied overnight at $4^{\circ} \mathrm{C}$. Membranes were stripped and reprobed with $\beta$-actin (Sigma) to confirm equal loading. The primary antibody was detected using a horseradish peroxidase-conjugated sheep anti-rabbit antibody and enhanced chemiluminescence (GE Health Care).
The films were subsequently scanned and band intensity was quantified using densitometry software (alphEaseFC) and expressed as relative optical density (ROD). For immunoprecipitation, $200 \mu \mathrm{g}$ of retinal protein was diluted with radioimmunoprecipitation assay buffer and incubated with $3 \mu \mathrm{g}$ of anti-Trx antibody. Samples were rocked at $4^{\circ} \mathrm{C}$ overnight followed by $40 \mu \mathrm{l}$ of agarose beads and rocked at $4^{\circ} \mathrm{C}$ for 4 hours. Final extract was boiled, processed and analyzed as described above. Membrane was probed with antibodies for ASK-1, TXNIP, or Trx for equal loading.

\section{Isolation of Retinal Vasculature}

Retinal vasculatures were isolated as described previously. ${ }^{2,21}$ Briefly, freshly enucleated eyes were fixed with $2 \%$ paraformaldehyde overnight. Retina cups were dissected, washed in phosphate-buffered saline and then incubated with $3 \%$ crude trypsin in $20 \mathrm{nmol} / \mathrm{L}$ Tris buffer, $\mathrm{pH} 8$, at $37^{\circ} \mathrm{C}$ for 2 hours. Vitreous and nonvascular cells were gently removed from the vasculature. The vasculature was soaked in several washes of $5 \%$ Triton X-100 to get rid of neuronal retina. The transparent vasculature was laid out on slides and used for TUNEL assay and acellular capillary examination.

\section{Determination of Degenerated (Acellular) Capillaries and Pericytes}

Retinal vasculature sections were stained with periodic acid-Schiff and hematoxylin. Acellular capillaries were quantified under microscope (400x) in a masked manner. Acellular capillaries were identified as capillary-sized vessel tubes having no nuclei anywhere along their length. Nuclei of pericytes were identified as being darker and smaller than endothelial cell nuclei and lie in the outer aspect of the capillary wall. Six animals were used for each group and the number of acellular capillaries and pericytes were counted in 10 fields of different areas of the mid-retina and calculated as the number $/ \mathrm{mm}^{2}$ of retinal area.

\section{TUNEL Assay of Isolated Vasculature}

The retinal vasculature was washed extensively in PBS and then fixed with $2 \%$ paraformaldehyde. The samples were incubated with terminal deoxynucleotidyl transferase enzyme followed by incubation with anti-digoxigenin conjugate. Positively labeled cells were identified in the whole retinal vasculature under AxioObserver.Z1 fluorescence microscope (Zeiss, Germany) with 400X magnification.

\section{Statistical Analysis}

The results were expressed as mean \pm SEM. Differences among experimental groups were evaluated by analysis of variance and the significance of differences between groups was assessed by the post hoc test (Fisher's PLSD) when indicated. Significance was defined as $P<0.05$. 


\section{Results}

Decomposing Peroxynitrite-Protected Ganglion Cells in NMDA-Injected Retinas

To examine the causal role of peroxynitrite in mediating RGC death, the neuroprotective effect of the specific peroxynitrite decomposition catalyst, FeTPPs, was determined in NMDA model after 1 day. Intravitreal injection of NMDA (40 nmol) induced sever RGC death as indicated by sevenfold increase of TUNEL-labeled cells (arrows) mainly in RGC and inner nuclear layer of the rat retina compared with NMLA controls. Co-treatment with FeTPPs (100 $\mu \mathrm{g} / \mathrm{eye}$ ) blocked RGC death in NMDA-injected animals after 1 day (Figure 1, A and D). Neuronal death was further confirmed, at that time point, by the absence of TUNEL-labeled cells within blood vessels stained with lectin (data not shown). In parallel, NMDA-induced neuronal damage was assessed histologically by counting

A

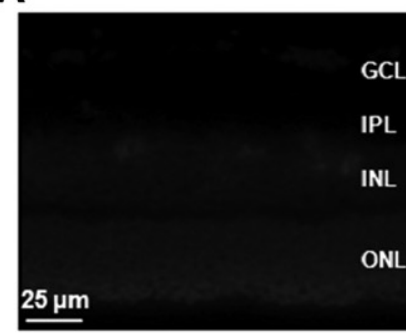

NMLA

B

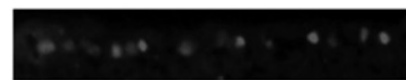

NMLA

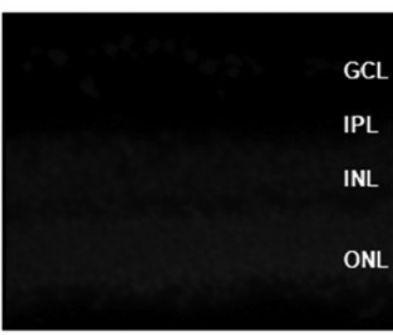

NMLA + FeTPPs

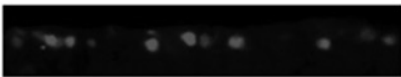

NMLA + FeTPPs

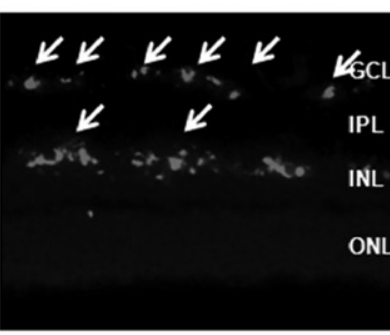

NMDA

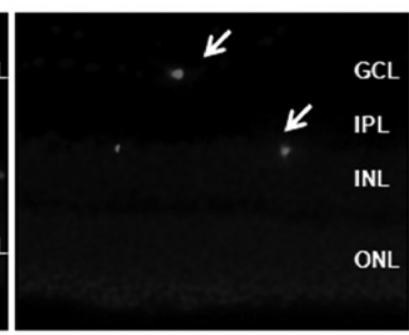

NMDA + FeTPPs

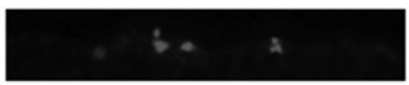

NMDA

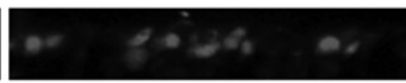

NMDA + FeTPPs

C

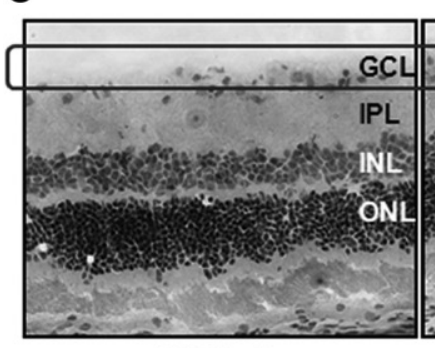

NMLA

D

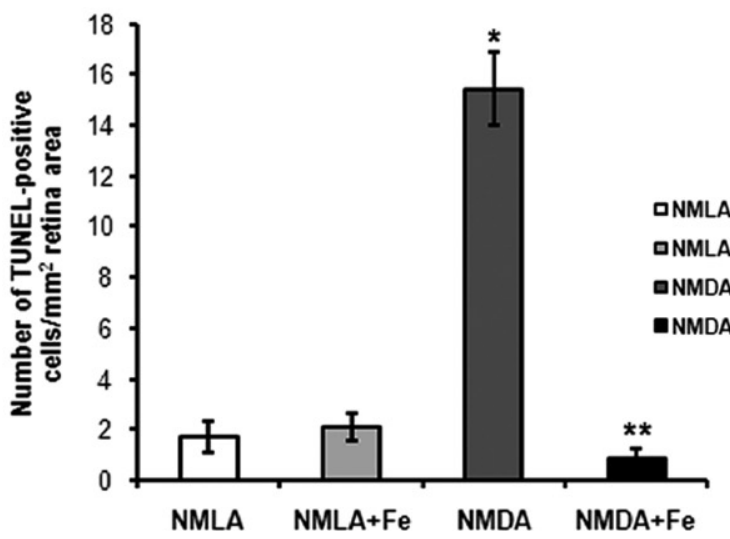

NMLA + FeTPPs

E

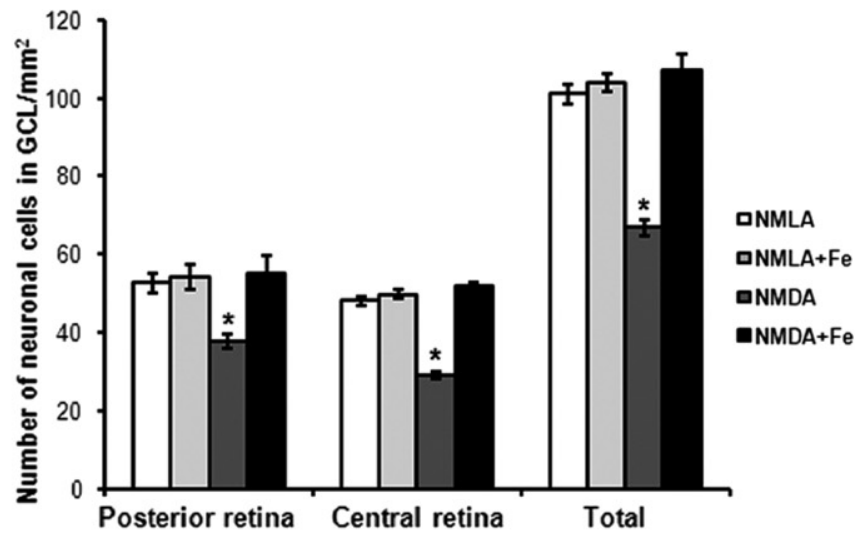

Figure 1. Decomposing peroxynitrite protected retinal ganglion cells in NMDA-injected retinas. A and D: Representative images and statistical analysis of TUNEL assay of rat retina after one day of the injection showing significant increase in number of TUNEL positive cells in NMDA-injected rats $(n=5-7, P<0.05)$, especially GCL as compared with NMLA controls or NMDA + FeTPPs. The number of TUNEL positive cells (arrows) were counted in four fields of different areas of each retina and calculated as the number $/ \mathrm{mm}^{2}$ of retinal area (magnification, $\times 200$ ). B: Representative images of rat retina sections stained with antiBrn-3a, specific RGC marker, showing reduced number of RGC in NMDA-injected retinas as compared with NMLA controls. C and E: Representative images and statistical analysis of rat retina sections stained with H\&E showing a reduction in number of GCL in NMDA-injected rats (magnification, $\times 200$ ) as compared with NMLA controls or NMDA + FeTPPs in central and posterior retina $(n=4-6, P<0.05)$. FeTPPs blocked all these effects in NMDA-injected retinas but did not affect NMLA control. There was a significant difference as compared with the rest of the groups at ${ }^{*} P<0.05$. There was a significant difference as compared with the control groups at ${ }^{* *} P<0.05$. GCL, ganglion cell layer; ONL, outer nuclear layer; IPL, inner plexiform layer; INL, inner nuclear layer. 
the number of neuronal cells in the GCL after 1 day of the injection (Figure 1, C and E). Intravitreal NMDA injections resulted in loss of $30 \%$ of cells in the GCL in the posterior regions of the retina and $40 \%$ of cells in GCL in the central regions of the retina. In addition, retina sections stained with Brn-3a, specific RGC marker, showed similar trend of reducing RGC number in NMDA-injected retinas as compared with NMLA control (Figure 1B). Co-treatment with FeTPPs (100 $\mu$ g/eye) prevented loss in ganglion cells and restored their number back to the control levels. Treatment with FeTPPs did not affect NMLA-injected controls.

\section{FeTPPs Blocked Nitrotyrosine in NMDA-Injected Retinas}

The efficiency of FeTPPs in decomposing peroxynitrite formation in NMDA-injected animals after 1 day was assessed by nitrotyrosine formation. Immunohistochemistry of nitrotyrosine, the footprint of peroxynitrite showed 1.8fold increases in NMDA-treated animals compared with NMLA controls. As shown in Figure 2, A and B, nitrotyrosine was immunolocalized mainly in the inner nuclear layer and RGC layer. Co-treatment with FeTPPs blocked nitrotyrosine formation in NMDA-injected animals but did not alter control NMLA-injected animals.

\section{FeTPPs Reduced TXNIP but Not Trx Expression in NMDA-Injected Retinas}

The expression of TXNIP, an endogenous Trx inhibitor, can be induced by oxidative stress. Real-time PCR anal- ysis showed threefold increase in mRNA levels of both TXNIP and Trx in NMDA-injected rat retinas after 1 day of the injection compared with NMLA controls. Co-treatment with FeTPPs reduced TXNIP mRNA levels to 1.5-fold but did not affect Trx level (Figure 3, A and B). In parallel, Western blot analysis of rat retina homogenates showed about 1.9-fold increase in TXNIP protein expression in NMDA-injected retinas compared with the NMLA controls (Figure 3C). Simultaneous injection of FeTPPs in NMDAinjected animals blocked this effect. Treatment with FeTPPs did not alter TXNIP expression in treated controls. These results suggest that intravitreal NMDA injection resulted in up-regulation of thioredoxin antioxidant system and that treatment with FeTPPs maintained high levels of antioxidant Trx but not the endogenous inhibitor TXNIP

\section{Increased TXNIP Binds Trx and Release ASK-1 from TRX-ASK-1 "Inhibitory Complex"}

Under resting conditions, Trx binds to and inhibits the apoptotic protein ASK-1 by forming Trx-ASK-1 "inhibitory complex." The interaction between Trx and ASK-1 in rats after 1 day of NMDA injection was investigated using immunoprecipitation assay. Our results showed that NMDA injection decreased the association between Trx and ASK-1 to only $35 \%$ of the basal level compared with NMLA controls (Figure $4 \mathrm{~A}$ ). Meanwhile, the association between Trx and TXNIP showed 1.7-fold increase in NMDA-injected rats compared with NMLA controls (Figure $4 \mathrm{~B})$. Co-treatment of animals with FeTPPs signifi-

A

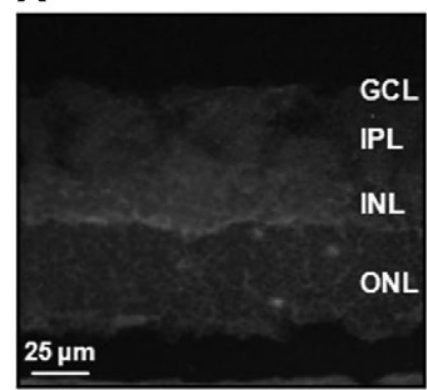

NMLA

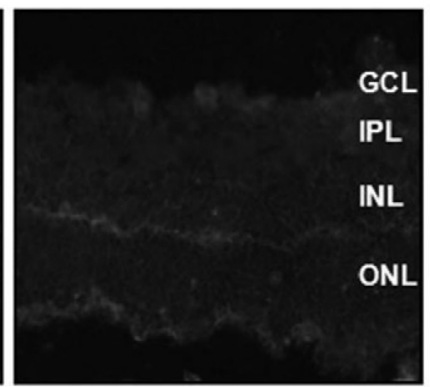

NMLA + FeTPPs

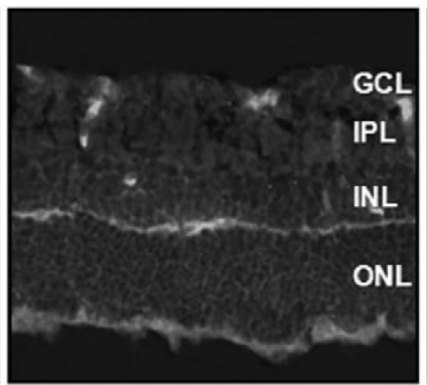

NMDA

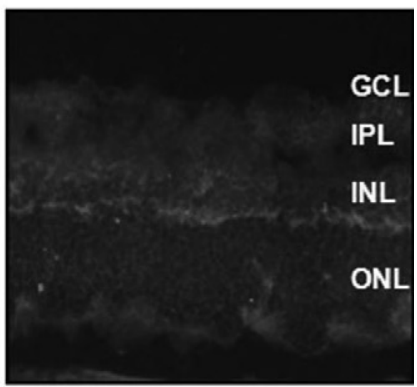

NMDA + FeTPPs

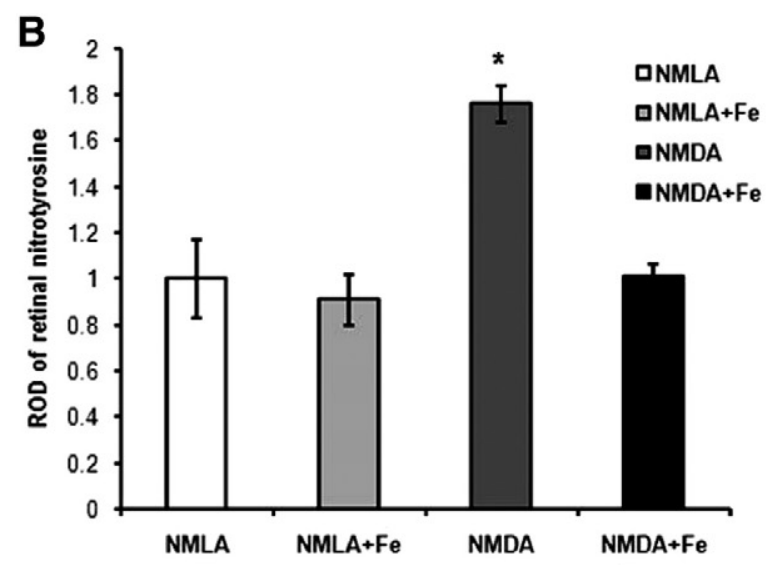

Figure 2. FeTPPs blocked nitrotyrosine in NMDA-injected retinas. A: Representative images showing NMDA-stimulated peroxynitrite formation in rat retina indicated by its footprint nitrotyrosine (magnification, $\times 200$ ) after one day of intravitreal NMDA injection. FeTPPs blocked this effect in NMDA-injected retinas but did not affect NMLA controls. B: Statistical analysis showed 1.8-fold increase in ROD of NMDAinjected retinas as compared with NMLA controls or NMDA + FeTPPs $(n=6)$. There was a significant difference as compared with the rest of the groups at ${ }^{*} P<0.05$. GCL, ganglion cell layer; ONL, outer nuclear layer; IPL, inner plexiform layer; INL, inner nuclear layer. 


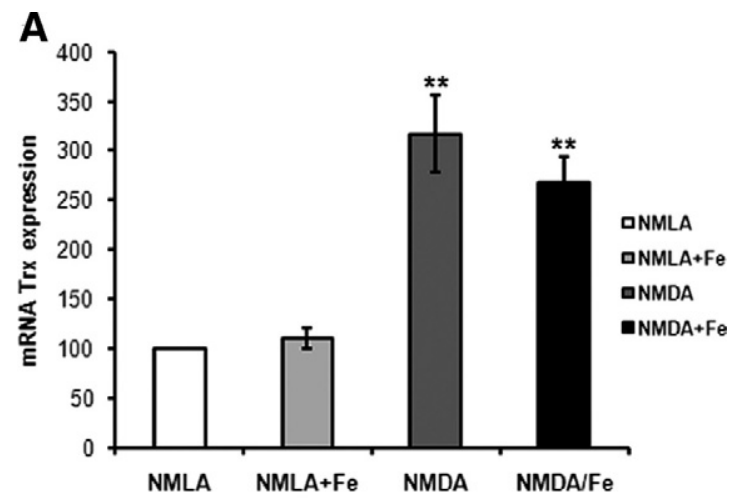

B

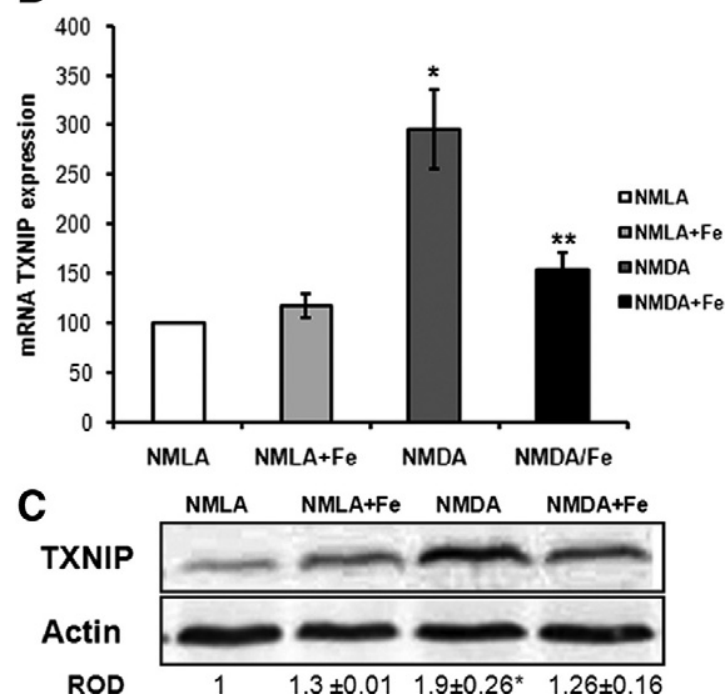

Figure 3. FeTPPs reduced NMDA-induced TXNIP but not Trx expression in NMDA-injected retinas. A: One day post NMDA injection, real-time PCR of retinal lysate showed a threefold increase in Trx expression in NMDAinjected retinas that was maintained by FeTPPs treatment as compared with NMLA controls $(n=5)$. B: Real-time PCR of retinal lysate showed a threefold increase in TXNIP expression in NMDA-injected retinas compared with NMLA controls $(n=5)$. C: Western blot of rat retinal lysates showed 1.9-fold increase in TXNIP in NMDA-injected retinas compared with NMLA control $(n=5-7)$. FeTPPs (100 $\mu \mathrm{g} /$ eye) blocked TXNIP expression in NMDAinjected rats but did not affect its expression in NMLA controls. There was a significant difference as compared with rest of the groups at ${ }^{*} P<0.05$. There was a significant difference as compared with controls at ${ }^{* *} P<0.05$.

cantly restored the association between Trx-ASK-1 (70\% of its basal level) and significantly reduced the interaction between Trx and TXNIP to 1.3-fold. Treatment with FeTPPs did not alter protein interaction in NMLA controls.

\section{FeTPPs Blocked ASK-1 Expression in Retinal Layers}

Next, we examined the expression and release of ASK-1 in rat retina after 1 day of NMDA injection using Western blot analysis. As shown in Figure 4C, NMDA injection caused 1.9-fold increase in ASK-1 expression in rat retina as compared with the NMLA controls. Although Western blot include proteins from all retinal cell types, the results were further confirmed and complemented with immunostaining assay. NMDA injection resulted in prominent immunolocalization of ASK-1 in the RGC and inner retinal layers as compared with NMLA controls (Figure 4D). Co-treatment with FeTPPs significantly reduced expression and localization of ASK-1 in NMDA-injected animals but not in treated NMLA controls.

\section{FeTPPs Blocked Activation of p38 MAPK Apoptotic Pathway}

ASK-1 has been shown to induce apoptosis by activation of p38 MAPK/JNK pathway. ${ }^{13}$ Therefore, we determined activation of p38 MAPK in NMDA-injected animals after 1 day. Western blot analysis showed 1.8-fold increase in p38 MAPK phosphorylation in NMDA-injected retinas as compared with NMLA controls. Apoptosis was further confirmed by the expression of cleaved PARP, a marker of energy failure. NMDA increased cleaved PARP expression by 2.3-fold in NMDA-injected animals compared with NMLA controls. Co-treatment with FeTPPs significantly reduced p38 MAPK activation and blocked cleaved PARP expression in NMDA-injected animals but did not alter the basal levels in NMLA controls (Figure 5, $A$ and $B$ ).

\section{FeTPPs Prevented Müller Glial Cell Activation in NMDA-Injected Retinas}

We next examine the protective action of decomposing peroxynitrite on glial activation. As shown in Figure $6 \mathrm{~A}$, NMDA-injected rats after 1 day showed a substantial increase in the intensity of GFAP immunoreactivity in the filaments of Müller cells that extended from the nerve fiber layer and inner plexiform layer into the outer nuclear layer $(\mathrm{ONL})$ of retina as compared with NMLA controls. Co-treatment of rats with FeTPPs blocked this effect in NMDA-injected rats but not in NMLA controls, suggesting that peroxynitrite play a causal role in causing glial activation following neuronal injury.

\section{FeTPPs Prevented NMDA-Induced Capillary Degeneration and Pericytes Loss}

Previous studies have demonstrated compromised vasculature following retinal neurodegeneration. 2,16 Therefore, we examined retinal vasculature 7 days after NMDA-induced neuronal injury and glial activation. A representative image of acellular capillaries stained with periodic acid-Schiff and hematoxylin was shown in Figure 6B. Acellular capillaries are demonstrated in Figure 6C using confocal microscopy of trypsin digest. All endothelial cells are labeled with isolectin (red) while collagen IV (green) labels vascular basement membrane. The circle superimposed on the merged image demonstrates a typical acellular capillary in retina (collagen IV positive but isolectin negative). Our results showed 2.6fold increase in the number of acellular capillaries and a $23 \%$ decrease in pericyte count in the NMDA-injected retinas compared with NMLA controls (Figure 6, D-F). Interestingly, NMDA exerted dose-dependent effect in causing capillary degeneration, as $80 \mathrm{nmol}$ of NMDA showed a 5.7-fold increase in the number of acellular capillaries as 
A

\section{IP by Trx}

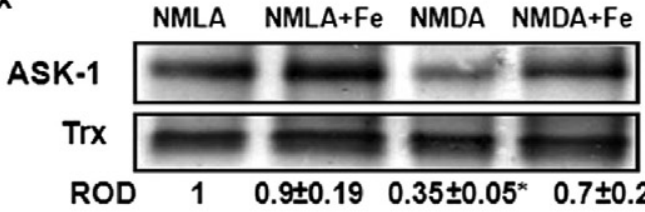

B

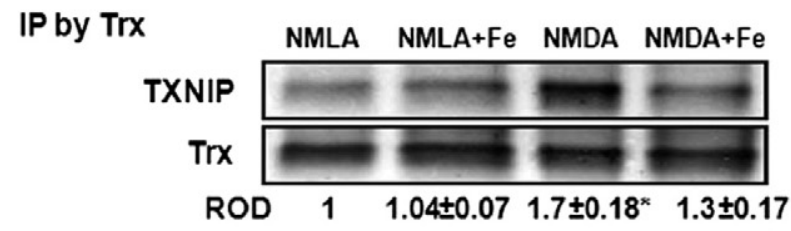

C

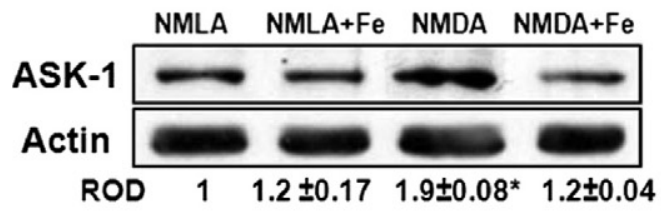

D

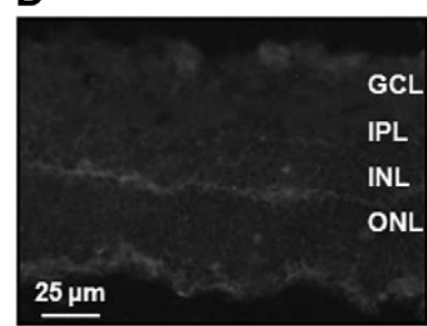

NMLA

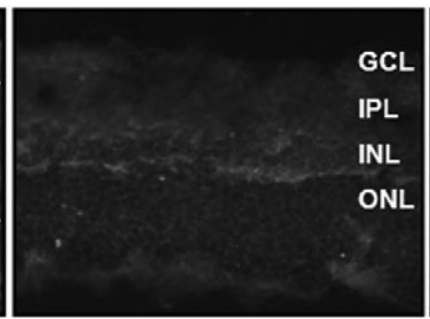

NMLA + FeTPPs compared with NMLA controls (Figure 6D). Moreover, TUNEL assay showed several positive cells in NMDA-injected animals suggesting that capillary degradation occurs through apoptosis (Figure 6). Co-treatment of rat reti-

A

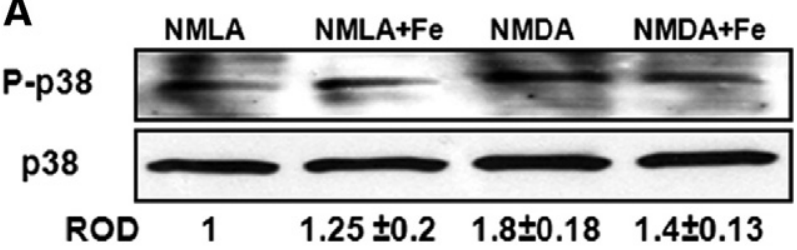

B

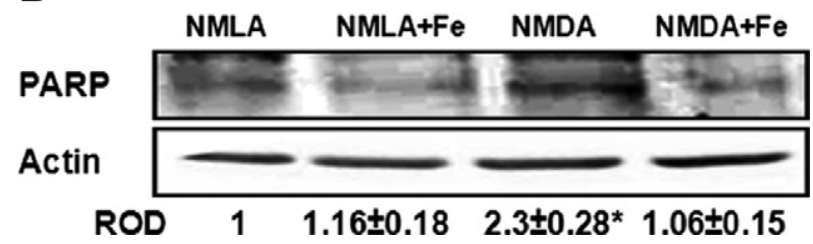

Figure 5. FeTPPs blocked activation of p38 MAPK apoptosis pathway. A: After one day of intravitreal NMDA injection, Western blot analysis showed 1.8-fold increase in $\mathrm{p} 38$ MAPK phosphorylation in rat retina lysate of NMDAinjected retinas compared with NMLA controls $(n=5-6)$. B: Western blot analysis of rat retina lysate showed a 2.3 -fold increase in cleaved PARP expression in NMDA-injected retinas compared with NMLA controls $(n=4)$. Treatment of animals with FeTPPs (100 $\mu \mathrm{g}$ /eye) reversed these effects in NMDA-injected retinas but did not affect NMLA controls. There was a significant difference as compared with rest of the groups at ${ }^{*} P<0.05$.
Figure 4. Increased TXNIP bind Trx and released ASK-1 from inhibitory complex. A: Immunoprecipitation of Trx and immunoblotting with ASK-1 showed $65 \%$ reduction in interaction between Trx and ASK-1 in NMDA-injected rats after one day as compared with NMLA controls $(n=4-5)$. B: Immunoprecipitation of Trx and immunoblotting with TXNIP showed a 1.7-fold increase in interaction between Trx and TXNIP in NMDA-injected retinas as compared with NMLA control $(n=4)$. C: Western blot analysis showed a 1.9-fold increase in ASK-1 expression in rat retina lysate of NMDA-injected retinas as compared with the NMLA controls ( $n=4-6$ ). D: Immunolocalization of ASK-1 in retinal ganglion cell and inner retinal layers in NMDA-injected retinas compared with NMLA controls (magnification, $\times 200$ ). FeTPPs blocked all these effects in NMDAinjected retinas but did not affect NMLA controls. There was a significant difference as compared with the rest of the groups at ${ }^{*} P<0.05$. GCL, ganglion cel layer; ONL, outer nuclear layer; IPL, inner plexiform layer; INL, inner nuclear layer.

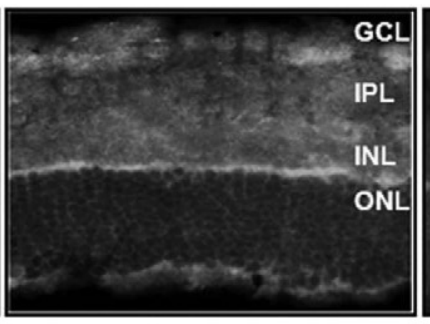

NMDA

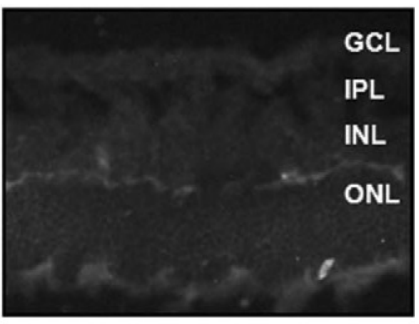

NMDA + FeTPPs

nas with FeTPPs restored the number of pericytes and decreased the number of acellular capillaries to the normal values of NMLA controls.

\section{Discussion}

The main findings of the current study using NMDA model': Decomposing peroxynitrite using FeTPPs prevents retinal ganglion cell loss and glial activation." Up-regulated peroxynitrite formation and TXNIP expression disrupt Trx-ASK-1 inhibitory complex leading to activation of ASK-1 apoptotic pathway. "I Initial neuroprotection effects of FeTPPs prevented long term vascular cell death and capillary degeneration. ${ }^{\mathrm{IV}}$ NMDA model can be useful tool to study glial activation and capillary degeneration, hallmarks of diabetic retinopathy in much shorter time.

Neuronal injury and death play an early and critical role in the pathogenesis of retinal neurodegenerative disorders, including glaucoma, diabetic retinopathy, uveitis, and retinal vein occlusion. ${ }^{1-6}$ In response to traumatic compression or vascular occlusion, excess glutamate is released to overstimulate the NMDA receptors leading to excessive $\mathrm{Ca}^{2+}$ influx, which in turn triggers formation of nitric oxide, accumulation of superoxides and peroxyni- 
A

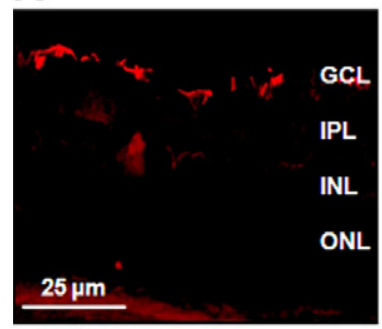

NMLA

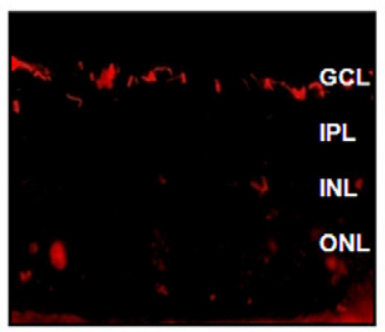

NMLA + FeTPPs

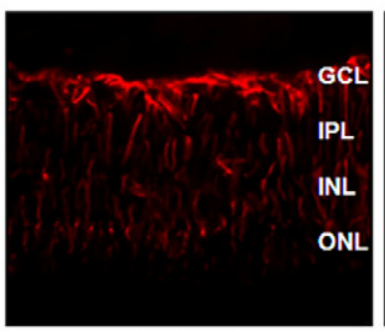

NMDA

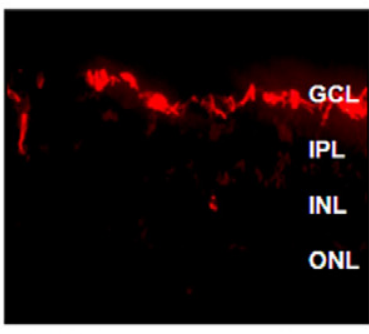

NMDA + FeTPPs
B

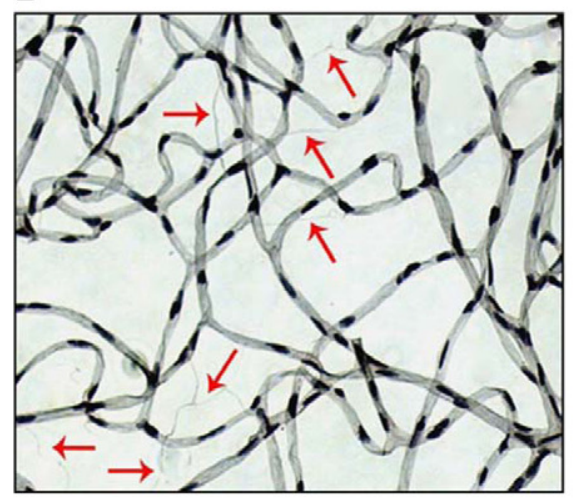

D

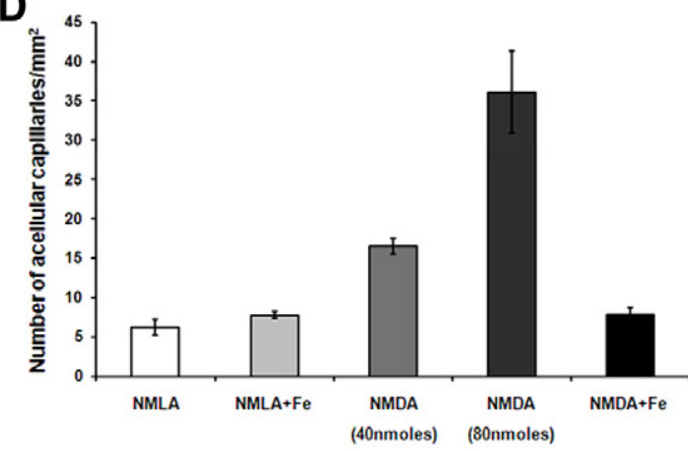

C
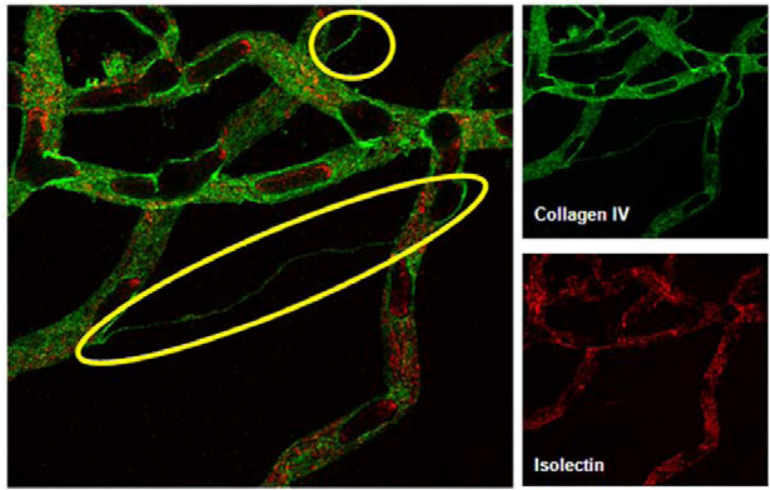

E

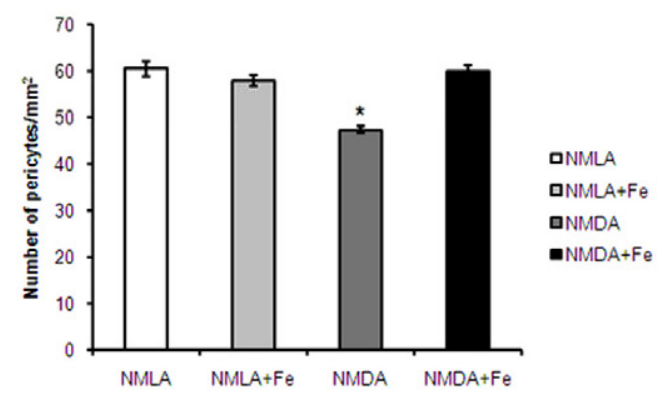

$\mathbf{F}$

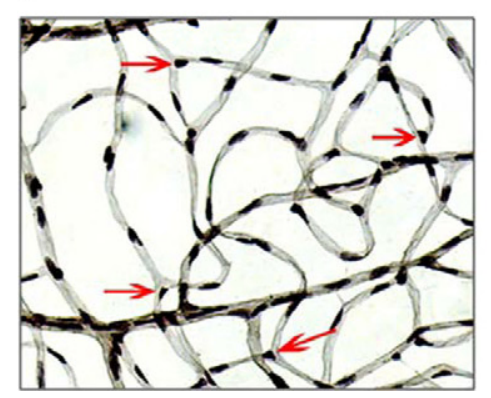

G

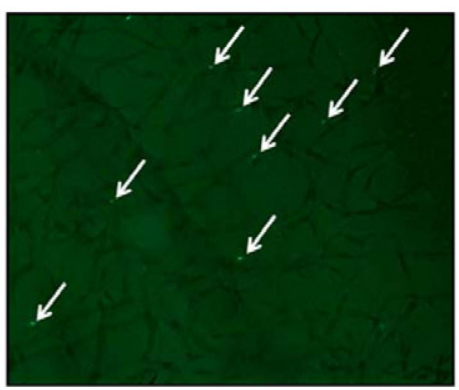

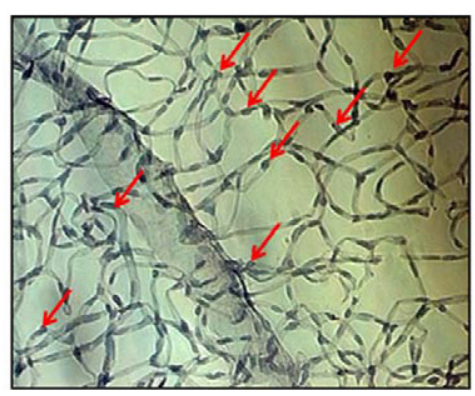

Figure 6. FeTPPs prevented NMDA-induced glial activation, capillary degeneration and pericytes loss. A: Representative images of GFAP immunofluorescence in various treatment groups after one day. In NMDA-injected retinas, the end feet of the Müller cells showed abundant GFAP immunofluorescence (red), and the radial processed stained intensely throughout both the inner and the outer retina (magnification, $\times 400$ ). Similar morphological changes were observed in $3-4$ animals. B: Representative image of trypsin-digest stained with periodic acid-Schiff and hematoxylin showing acellular capillaries (arrows) in NMDA-injected rats after seven days (magnification, $\times 400$ ). C: Representative images of acellular capillaries using confocal microscopy of trypsin digest. All endothelial cells are labeled with isolectin (red) while collagen IV (green) labels vascular basement membrane. The circle superimposed on the merged image demonstrates a typical acellular capillary in retina (collagen IV positive but isolectin negative). D: Statistical analysis showing 2.6-fold increase in number of acellular capillaries in NMDA-injected retinas compared with NMLA controls or NMDA + FeTPPs $(n=4-6)$. E-F: Statistical analysis and representative image of retinal trypsin-digest stained with periodic acid-Schiff (PASH) and Hematoxylin showing pericyte cells (indicated by red arrows) in NMDA-injected rats showing a significant decrease in pericytes count in NMDA-injected retinas compared with NMLA-controls or NMDA + FeTPPs $(\mathrm{n}=4-6)(\mathrm{magnification}$, $\times 400)$. These effects were blocked by FeTPPs in NMDA-injected retinas but did not alter NMLA-controls. G: Representative image of retinal trypsin digest stained with TUNEL (left) showing that vascular cell death occurs by apoptosis as indicated by TUNEL positive nuclei (white arrows) in endothelial cells (red arrows) ( $400 \times$ magnification). There was significant difference against the controls and NMDA + FeTPPs groups at ${ }^{*} P<0.05$. There was significant difference against the rest of the groups at $\# P<0.05$. GCL, ganglion cell layer; IPL, inner plexiform layer; INL, inner nuclear layer; ONL, outer nuclear layer. 
trite, and eventually cause RGC death. ${ }^{1,7,9,22}$ Therefore, an NMDA model was used to examine the causal role of peroxynitrite in mediating neuronal cell death and to further assess its subsequent effects on glial activation and retinal vasculature. Indeed, our results showed significant increase in peroxynitrite formation as indicated by 1.8-fold increase in nitrotyrosine formation in NMDA-injected rats that was blocked by co-treatment with the peroxynitrite decomposition catalyst FeTPPs. The neuroprotective effects of FeTPPs were evident by significant reduction of TUNEL-positive nuclei in GCL, restoration of neuronal cell count in GCL at both central and posterior retina back to the normal level, as well as restoration of RGC count in retina sections stained with anti-Brn-3a, a specific RGC marker. Of note, the number of RGC cells (Brn-3a positive) represented about $\sim 60 \%$ of the neuronal cells in GCL, which is consistent with the fact that displaced amacrine cells constitute $\sim 40 \%$ of GCL. ${ }^{23}$ These results establish a causal role of peroxynitrite in mediating retinal neurotoxicity and lend further support to previous reports showing protective effects of blocking peroxynitrite indirectly using the NOS inhibitor L-NAME, iNOS inhibitor aminoguanidine, or the superoxides and hydroxyl radicals scavenger TEMPOL in NMDA or ischemia/reperfusion model. ${ }^{1,2,24,25}$ The neuroprotective effects of FeTPPs were screened after 1 day of NMDA injection, a time point at which, RGC death will peak then plateau afterward. ${ }^{1,26}$ Indeed, peroxynitrite decomposition catalysts, such as FeTPPs and FP15 have been proven effective in reducing peroxynitrite-mediated insults in models of ischemic retinopathy, diabetic retinopathy, and neuropathy. ${ }^{3,20,27-30}$

To elucidate the molecular mechanism of the neuroprotective actions of FeTPPs, we next examined the expression of the antioxidant and antiapoptotic Trx system in NMDA-injected retinas. The activity of Trx and its ability to scavenge free radicals is negatively regulated by binding to TXNIP, which is known to be enhanced by $\mathrm{Ca}^{2+}$ influx. ${ }^{14}$ Certainly, NMDA stimulates $\mathrm{Ca}^{2+}$ influx, and it was not surprising to observe significant increases of TXNIP mRNA and protein expression (three- and twofold, respectively) in response to NMDA. In contrast, NMDA induced Trx mRNA (threefold) possibly as self-defense, which was maintained by treatment with FeTPPs for the short term (1 day). Therefore, the increased binding of TXNIP to Trx may account for the functional inhibition of Trx activity and mediate oxidative stress. Of note, longterm studies ( 7 days) revealed significant reduction of Trx mRNA (50\%) in NMDA-injected animals (data not shown). A recent study demonstrated that inhibiting TXNIP using calcium channel blockers can prevent cardiac apoptosis. ${ }^{31}$ This is of importance as reduced Trx has also been shown to play a key role in regulating apoptosis pathway by binding to ASK-1 through redox active site of Trx. ${ }^{32}$ Thus, inactivation of Trx through oxidation or interaction with TXNIP leads to release and activation of the apoptotic ASK-1. Indeed, our analyses showed up-regulated TXNIP expression was associated with 1.7-fold increase in its interaction with Trx and $~ 65 \%$ reduction in interaction between Trx and ASK1 (Figure 4). Western blot analysis confirmed significant increases 1.9-fold in ASK-1 expression in NMDA-injected animals that was localized mainly in the ganglion cell layer and inner retinal layers. Although, Western blot include proteins from all retinal cell types, the distinctive changes in protein expression might be attributed to the acute nature of the neurotoxic NMDA model. Immuno-colocalization studies of ASK-1 with RGC marker (Brn-3a), but not isolectin-B4 showed prominent staining in $\mathrm{GCL}$ and inner retina layers (data not shown). These results complement the proposed role of peroxynitrite in releasing ASK-1 and causing RGC death in NMDA model. Co-treatment with FeTPPs inhibited the association of Trx and TXNIP, restored the association between Trx and ASK-1 and reduced ASK-1 expression. In support, several studies demonstrated that restoring Trx activity can prevent RGC death and apoptosis in aging liver cells or cardiomyocytes. ${ }^{11,33-35}$ However, our study is the first one to demonstrate a causal role of peroxynitrite in increasing TXNIP expression, altering thioredoxin system and activating ASK-1 proapoptotic pathway leading to RGC death in NMDA model.

We next evaluated whether the increases in ASK-1 triggered apoptosis in NMDA-injected rat retinas. Indeed, our results showed significant increases in phosphorylated p38 MAPK and cleaved PARP expression in NMDA-injected animals. In agreement, NMDA-induced activation of p38 MAPK and PARP has been well documented and linked to RGC death in vitro and in vivo. ${ }^{36-40}$ In addition, the neuroprotective actions of inhibiting p38 MAPK using SB203580 has been demonstrated in NMDA-injected rats, ${ }^{37}$ as well as in hippocampal neuronal cultures ${ }^{41}$ and in cortical cell cultures. ${ }^{42}$ Our results showed that the neuroprotective effects of FeTPPs was associated with clear suppression of p38 MAPK activation and cleaved PARP expression. In support, previous studies demonstrated similar protective actions of decomposing peroxynitrite in models of diabetic retinopathy, oxygen-induced retinopathy, or cardiomyopathy. ${ }^{3,20,35,43}$

Histological studies demonstrate remarkable activation of retinal glial cells including astrocytes and Muller cells in human and experimental models of glaucoma, diabetic retinopathy and retinal ischemia. ${ }^{18,44-47}$ In agreement, our results confirmed glial activation as evident by prominent localization of the gliofilaments GFAP in NMDA-injected retinas that was blocked by treatment with FeTPPs. Because of glial activation, previous studies documented increased light scatter clinically ${ }^{45}$ and significant increases in inflammatory mediators such as interleukin- $\beta$, tumor necrosis factor- $\alpha$, nuclear factor $\kappa \mathrm{B}$, and ICAM expression experimentally ${ }^{47-49}$ that can play a major role in not only sustaining the neuronal cell death but to extend the damage to retinal vasculature. Therefore, we examined the long term effects of the initial neuroglial injury on retinal capillary degeneration, 7 days after NMDA injection. Our results demonstrated significant effects of NMDA injection on increasing capillary degeneration and pericytes loss as well as several TUNEL-labeled cells in retinal capillaries that were blocked by co-treatment with FeTPPs. Based on the time course of degeneration of both the neuronal and vascular components, significant neuronal loss in the retina pre- 
cedes capillary degeneration in response to NMDA. This observation is in agreement with the notion that early and gradual loss of neurons may be a primary abnormality that gives rise to vascular changes seen in diabetic retinopathy, ischemic retinopathy, and models of ischemia reperfusion.

The observed vascular cell death in response to NMDA could be attributed to several mechanisms including direct effects of activating NMDA receptors leading to peroxynitrite formation and ensuing apoptosis in endothelial cells, ${ }^{50,51}$ neuroglia injury and the release of inflammatory mediators and/or possible increases in TXNIP and activation of ASK-1 apoptotic pathway. In support, our results, in NMDA model, showed neuro- and vascular protective actions of FeTPPs, which parallel its neuroprotective action in models of diabetic and ischemic retinopathy. Furthermore, co-localization studies showed that peroxynitrite formation can be detected in both neuronal and vascular areas of the retina. Although we did not examine the direct role of increased TXNIP expression in activating apoptotic signal in retinal capillaries, previous studies demonstrated that overexpression of TXNIP augment the inflammatory actions of tumor necrosis factor$\alpha$-mediated activation of p38 MAPK and apoptosis in vascular endothelial cells. ${ }^{52,53}$ In addition, activation of PARP in retina contributes to vascular cell death and the formation of pericyte ghosts and acellular capillaries in vitro and in vivo. ${ }^{20,28,44,48}$ The significance of capillary degeneration that followed neuroglial injury in NMDA model bring attention to ischemia and impaired reperfusion that take place in traumatic optic neuropathy and glaucoma. ${ }^{54}$

In summary, retinas injected with NMDA can provide a good tool to study ROS generation, RGC cell death, glial activation, and capillary degeneration. Although having different etiology, these endpoints have a lot in common with some of the hallmarks of diabetic retinopathy. Morphologically, glial activation observed 1 day after NMDA injection is similar to that observed 2-3 months in diabetic retinopathy. Moreover, degenerated capillaries that detected after 7 days in NMDA model seem comparable to acellular capillaries detected $6-8$ months in diabetic retinopathy. Retinas from rodents after NMDA injection show biochemical alterations that also are seen in diabetes, including increased formation of peroxynitrite and expression of apoptotic markers p-p38 MAPK and PARP. Based on these similarities between NMDA model and diabetic retinopathy, we postulate that the retinal NMDA model may be used as an acute model for screening therapeutic approaches to inhibit retinal neuronal death, glial activation, and capillary degeneration in diabetic retinopathy and other retinopathies.

\section{Acknowledgment}

We thank Dr. Tom Gardiner (Queens University Belfast, UK) for his expertise and advice on trypsine digest.

\section{References}

1. El-Remessy AB, Khalil IE, Matragoon S, Abou-Mohamed G, Tsai NJ Roon P, Caldwell RB, Caldwell RW, Green K, Liou GI: Neuroprotective effect of (-)Delta9-tetrahydrocannabinol and cannabidiol in N-methylD-aspartate-induced retinal neurotoxicity: involvement of peroxynitrite. Am J Pathol 2003, 163:1997-2008

2. Zheng L, Gong B, Hatala DA, Kern TS: Retinal ischemia and reperfusion causes capillary degeneration: similarities to diabetes. Invest Ophthalmol Vis Sci 2007, 48:361-367

3. Ali TK, Matragoon S, Pillai BA, Liou GI, El-Remessy AB: Peroxynitrite mediates retinal neurodegeneration by inhibiting NGF survival signal in experimental and human diabetes. J Diabetes 2008, 57:889-898

4. El-Remessy AB, Tang Y, Zhu G, Matragoon S, Khalifa Y, Liu EK, Liu JY, Hanson E, Mian S, Fatteh N, Liou GI: Neuroprotective effects of cannabidiol in endotoxin-induced uveitis: critical role of p38 MAPK activation. Mol Vis 2008, 14:2190-2203

5. Schmidt KG, Bergert $H$, Funk RH: Neurodegenerative diseases of the retina and potential for protection and recovery. Curr Neuropharmacol 2008, 6:164-178

6. Osborne NN, Ugarte M, Chao M, Chidlow G, Bae JH, Wood JP, Nash MS: Neuroprotection in relation to retinal ischemia and relevance to glaucoma. Surv Ophthalmol 1999, 43(Suppl 1):S102-S128

7. Lam TT, Abler AS, Kwong JMK, Tso MOM: N-methyl-D-aspartate (NMDA)-induced apoptosis in rat retina. Invest Ophthalmol Vis Sci 1999, 40:2391-2397

8. Hirooka K, Kourennyi DE, Barnes S: Calcium channel activation facilitated by nitric oxide in retinal ganglion cells. J Neurophysiol 2000 83:198-206

9. Dreyer EB: A proposed role for excitotoxicity in glaucoma. J Glaucoma 1998, 7:62-67

10. Gauntt CD, Ohira A, Honda O, Kigasawa K, Fujimoto T, Masutani H, Yodoi J, Honda Y: Mitochondrial induction of adult $T$ cell leukemia derived factor (ADF/hTx) after oxidative stresses in retinal pigment epithelial cells. Invest Ophthalmol Vis Sci 1994, 35:2916-2923

11. Munemasa Y, Ahn JH, Kwong JM, Caprioli J, Piri N: Redox proteins thioredoxin 1 and thioredoxin 2 support retinal ganglion cell survival in experimental glaucoma. Gene Ther 2009, 16:17-25

12. Holmgren A: Thioredoxin structure and mechanism: conformational changes on oxidation of the active-site sulfhydryls to a disulfide. Structure 1995, 3:239-243

13. Ichijo H, Nishida E, Irie K, ten Dijke P, Saitoh M, Moriguchi T, Takagi M, Matsumoto K, Miyazono K, Gotoh Y: Induction of apoptosis by ASK1, a mammalian MAPKKK that activates SAPK/JNK and p38 signaling pathways. Science 1997, 275:90-94

14. Yamanaka H, Maehira F, Oshiro M, Asato T, Yanagawa Y, Takei H, Nakashima $Y$ : A possible interaction of thioredoxin with VDUP1 in HeLa cells detected in a yeast two-hybrid system. Biochem Biophys Res Commun 2000, 271:796-800

15. Nishiyama A, Matsui M, Iwata S, Hirota K, Masutani $H$, Nakamura $H$ Takagi $Y$, Sono $H$, Gon $Y$, Yodoi J: Identification of thioredoxinbinding protein-2/vitamin $\mathrm{D}(3)$ up-regulated protein 1 as a negative regulator of thioredoxin function and expression. J Biol Chem 1999, 274:21645-21650

16. Fletcher EL, Phipps JA, Wilkinson-Berka JL: Dysfunction of retinal neurons and glia during diabetes. Clin Exp Optom 2005, 88:132-145

17. Lam DY, Kaufman PL, Gabelt BT, To EC, Matsubara JA: Neurochemical correlates of cortical plasticity after unilateral elevated intraocular pressure in a primate model of glaucoma. Invest Ophthalmol Vis Sci 2003, 44:2573-2581

18. Nakazawa T, Takahashi H, Nishijima K, Shimura M, Fuse N, Tamai M, Hafezi-Moghadam A, Nishida K: Pitavastatin prevents NMDA-induced retinal ganglion cell death by suppressing leukocyte recruitment. J Neurochem 2007, 100:1018-1031

19. Siliprandi R, Canella R, Carmignoto G, Schiavo N, Zanellato A, Zanoni R, Vantini G: N-methyl-D-aspartate-induced neurotoxicity in the adult rat retina. Vis Neurosci 1992, 8:567-573

20. Abdelsaid MA, Pillai BA, Matragoon S, Prakash R, Al-Shabrawey M, El-Remessy AB: Early intervention of tyrosine nitration prevents vasoobliteration and neovascularization in ischemic retinopathy. $\mathrm{J}$ Pharmacol Exp Ther 2010, 332:125-134

21. Stitt AW, Li YM, Gardiner TA, Bucala R, Archer DB, Vlassara H: Advanced glycation end products (AGEs) co-localize with AGE re- 
ceptors in the retinal vasculature of diabetic and of AGE-infused rats. Am J Pathol 1997, 150:523-531

22. Kashii S, Mandai M, Kikuchi M, Honda Y, Tamura Y, Kaneda K, Akaike A: Dual actions of nitric oxide in N-methyl-D-aspartate receptor-mediated neurotoxicity in cultured retinal neurons. Brain Res 1996, 711:93-101

23. Perry VH, Henderson Z, Linden R: Postnatal changes in retinal ganglion cell and optic axon populations in the pigmented rat. J Comp Neurol 1983, 219:356-368

24. Morizane C, Adachi K, Furutani I, Fujita Y, Akaike A, Kashii S, Honda $Y$ : N(omega)-nitro-L-arginine methyl ester protects retinal neurons against $\mathrm{N}$-methyl-D-aspartate-induced neurotoxicity in vivo. Eur J Pharmacol 1997, 328:45-49

25. Neufeld AH, Sawada A, Becker B: Inhibition of nitric-oxide synthase 2 by aminoguanidine provides neuroprotection of retinal ganglion cells in a rat model of chronic glaucoma. Proc Natl Acad Sci USA 1999, 96:9944-9948

26. Ito $Y$, Shimazawa M, Inokuchi $Y$, Fukumitsu H, Furukawa S, Araie M, Hara $\mathrm{H}$ : Degenerative alterations in the visual pathway after NMDAinduced retinal damage in mice. Brain Res 2008, 1212:89-101

27. El-Remessy AB, Al-Shabrawey M, Platt DH, Bartoli M, Behzadian MA, Ghaly N, Tsai N, Motamed K, Caldwell RB: Peroxynitrite mediates VEGF's angiogenic signal and function via a nitration-independent mechanism in endothelial cells. FASEB J 2007, 10:2528-2539

28. El-Remessy AB, Bartoli M, Platt DH, Fulton D, Caldwell RB: Oxidative stress inactivates VEGF survival signaling in retinal endothelial cells via PI 3-kinase tyrosine nitration. J Cell Sci 2005, 118:243-252

29. Obrosova IG, Mabley JG, Zsengeller Z, Charniauskaya T, Abatan OI, Groves JT, Szabo C: Role for nitrosative stress in diabetic neuropathy: evidence from studies with a peroxynitrite decomposition catalyst. FASEB J 2005, 19:401-403

30. Drel VR, Pacher P, Vareniuk I, Pavlov I, Inytska O, Lyzogubov VV, Tibrewala J, Groves JT, Obrosova IG: A peroxynitrite decomposition catalyst counteracts sensory neuropathy in streptozotocin-diabetic mice. Eur J Pharmacol 2007, 569:48-58

31. Chen J, Cha-Molstad H, Szabo A, Shalev A: Diabetes induces and calcium channel blockers prevent cardiac expression of proapoptotic thioredoxin-interacting protein. Am J Physiol Endocrinol Metab 2009, 296:E1133-E1139

32. Liu H, Zhang H, Iles KE, Rinna A, Merrill G, Yodoi J, Torres M, Forman HJ: The ADP-stimulated NADPH oxidase activates the ASK-1/MKK4/ JNK pathway in alveolar macrophages. Free Radic Res 2006, 40:865-874

33. Inomata $\mathrm{Y}$, Nakamura $\mathrm{H}$, Tanito M, Teratani A, Kawaji T, Kondo N, Yodoi J, Tanihara H: Thioredoxin inhibits NMDA-induced neurotoxicity in the rat retina. J Neurochem 2006, 98:372-385

34. Hsieh CC, Papaconstantinou J: Thioredoxin-ASK1 complex levels regulate ROS-mediated p38 MAPK pathway activity in livers of aged and long-lived Snell dwarf mice. FASEB J 2006, 20:259-268

35. Zhang H, Tao L, Jiao X, Gao E, Lopez BL, Christopher TA, Koch W, Ma XL: Nitrative thioredoxin inactivation as a cause of enhanced myocardial ischemia/reperfusion injury in the aging heart. Free Radic Biol Med 2007, 43:39-47

36. Nicole O, Ali C, Docagne F, Plawinski L, MacKenzie ET, Vivien D, Buisson A: Neuroprotection mediated by glial cell line-derived neurotrophic factor: involvement of a reduction of NMDA-induced calcium influx by the mitogen-activated protein kinase pathway. J Neurosci 2001, 21:3024-3033

37. Manabe S, Lipton SA: Divergent NMDA signals leading to proapoptotic and antiapoptotic pathways in the rat retina. Invest Ophthalmol Vis Sci 2003, 44:385-392

38. Harper SJ, LoGrasso P: Signalling for survival and death in neurones: the role of stress-activated kinases. JNK and p38. Cell Signal 2001, 13:299-310

39. Pieper AA, Blackshaw S, Clements EE, Brat DJ, Krug DK, White AJ, Pinto-Garcia P, Favit A, Conover JR, Snyder SH, Verma A: Poly(ADPribosyl)ation basally activated by DNA strand breaks reflects glutamate-nitric oxide neurotransmission. Proc Natl Acad Sci USA 2000, 97:1845-1850

40. Li Y, Schlamp CL, Poulsen GL, Jackson MW, Griep AE, Nickells RW: p53 regulates apoptotic retinal ganglion cell death induced by $\mathrm{N}$ methyl-D-aspartate. Mol Vis 2002, 8:341-350

41. Molz S, Decker H, Dal-Cim T, Cremonez C, Cordova FM, Leal RB, Tasca Cl: Glutamate-induced toxicity in hippocampal slices involves apoptotic features and p38 MAPK signaling. Neurochem Res 2008, 33:27-36

42. Ko HW, Han KS, Kim EY, Ryu BR, Yoon WJ, Jung YK, Kim SU, Gwag $\mathrm{BJ}$ : Synergetic activation of $\mathrm{p} 38$ mitogen-activated protein kinase and caspase-3-like proteases for execution of calyculin A-induced apoptosis but not $\mathrm{N}$-methyl-d-aspartate-induced necrosis in mouse cortical neurons. J Neurochem 2000, 74:2455-2461

43. Levrand S, Pacher P, Pesse B, Rolli J, Feihl F, Waeber B, Liaudet L: Homocysteine induces cell death in $\mathrm{H} 9 \mathrm{C} 2$ cardiomyocytes through the generation of peroxynitrite. Biochem Biophys Res Commun 2007, 359:445-450

44. Drel VR, Xu W, Zhang J, Kador PF, Ali TK, Shin J, Julius U, Slusher B, El-Remessy AB, Obrosova IG: Poly(ADP-ribose)polymerase inhibition counteracts cataract formation and early retinal changes in streptozotocin-diabetic rats. Invest Ophthalmol Vis Sci 2009, 50:1778-1790

45. Wang L, Cioffi GA, Cull G, Dong J, Fortune B: Immunohistologic evidence for retinal glial cell changes in human glaucoma. Invest Ophthalmol Vis Sci 2002, 43:1088-1094

46. Mizutani M, Gerhardinger C, Lorenzi M: Muller cell changes in human diabetic retinopathy. Diabetes 1998, 47:445-449

47. Laabich A, Li G, Cooper NG: Enhanced expression of TNF-R1 protein in NMDA-mediated cell death in the retina. Brain Res Mol Brain Res 2002, 109:239-246

48. Zheng L, Szabo C, Kern TS: Poly(ADP-ribose) polymerase is involved in the development of diabetic retinopathy via regulation of nuclear factor-kappaB. Diabetes 2004, 53:2960-2967

49. Joussen AM, Poulaki V, Le ML, Koizumi K, Esser C, Janicki H, Schraermeyer U, Kociok N, Fauser S, Kirchhof B, Kern TS, Adamis AP: A central role for inflammation in the pathogenesis of diabetic retinopathy. FASEB J 2004, 18:1450-1452

50. Sharp CD, Hines I, Houghton J, Warren A, Jackson TH, Jawahar A Nanda A, Elrod JW, Long A, Chi A, Minagar A, Alexander JS: Glutamate causes a loss in human cerebral endothelial barrier integrity through activation of NMDA receptor. Am J Physiol Heart Circ Physiol 2003, 285:H2592-H2598

51. Sharp CD, Houghton J, Elrod JW, Warren A, Jackson TH, Jawahar A, Nanda A, Minagar A, Alexander JS: N-methyl-D-aspartate receptor activation in human cerebral endothelium promotes intracellular oxidant stress. Am J Physiol Heart Circ Physiol 2005, 288:H1893-H1899

52. Yamawaki H, Pan S, Lee RT, Berk BC: Fluid shear stress inhibits vascular inflammation by decreasing thioredoxin-interacting protein in endothelial cells. J Clin Invest 2005, 115:733-738

53. Chen CL, Lin CF, Chang WT, Huang WC, Teng CF, Lin YS: Ceramide induces p38 MAPK and JNK activation through a mechanism involving a thioredoxin-interacting protein-mediated pathway. Blood 2008, 111:4365-4374

54. Flammer J, Mozaffarieh M: What is the present pathogenetic concept of glaucomatous optic neuropathy? Surv Ophthalmol 2007, 52(Suppl 2):S162-S173 